\title{
Imprimer La Lumiere - 3D Printing Bioluminescence for Architectural Materiality
}

\author{
Mette Ramsgaard Thomsen ${ }^{1(\bigotimes)}$, Martin Tamke ${ }^{1}$, Aurelie Mosse ${ }^{2}$, \\ Jakob Sieder-Semlitsch ${ }^{1}$, Hanae Bradshaw ${ }^{2}$, Emil Fabritius Buchwald ${ }^{1}$, \\ and Maria Mosshammer ${ }^{3}$ \\ ${ }^{1}$ CITA, Royal Danish Academy, Copenhagen, Denmark \\ \{mette. thomsen, martin.tamke, jsie, efab\}@kglakademi.dk \\ 2 ENSAD, Paris, France \\ \{aurelie.mosse, hanae.bradshaw\} @ensad.fr \\ 3 University of Copenhagen, Copenhagen, Denmark \\ maria.mosshammer@bio.ku.dk
}

\begin{abstract}
Imprimer la Lumière' examines the making of a bioluminescent micro architecture. The project positions itself inside a sustainability agenda. By exploring the use of light-emitting bacteria as a material for architecture it asks what are the concepts, methods and technologies needed for designing with living materials. The project devises new means by which to design with the luminescent vibrio fischeri bacteria in a $3 \mathrm{D}$ printing manufacturing process based on extrusion principles. By combining the study of these living organisms and their appropriation through advanced robot-controlled 3D printing technologies, we establish a conceptual, material and technological framework for a bio-controlled bacteria growth and 3D extrusion process and a printable material based on agarose and gelatine.
\end{abstract}

Keywords: New materiality $\cdot$ Architecture $\cdot$ Bio-design $\cdot$ Robotic fabrication · Bioluminescence $\cdot 3 \mathrm{D}$ printing

\section{Introduction}

As we enter an era of resource scarcity, architecture and design communities are urgently rethinking their material practices. New conceptual frameworks allow us to consider a bio-based material paradigm as an inspirational model (biomimetics), a co-worker (biodesign) or a technological platform (bio-technology) allowing to reprogram the living (Collet 2013). Whether natural or synthetic, the development of biological manufacturing processes plays an increasing role in this context (Franklin and Till 2018; Terranova and Tromble 2017; Brayer and Zeitoun 2019), leading to the conceptualisation of a metabolic-driven material paradigm that challenges the perception of our surroundings as inert to something essentially living (Ramsgaard Thomsen 2019) (Fig. 1).

'Imprimer la Lumière' is an interdisciplinary research enquiry sitting at the intersection of architecture, design and microbiology exploring the use of light-emitting bacteria 


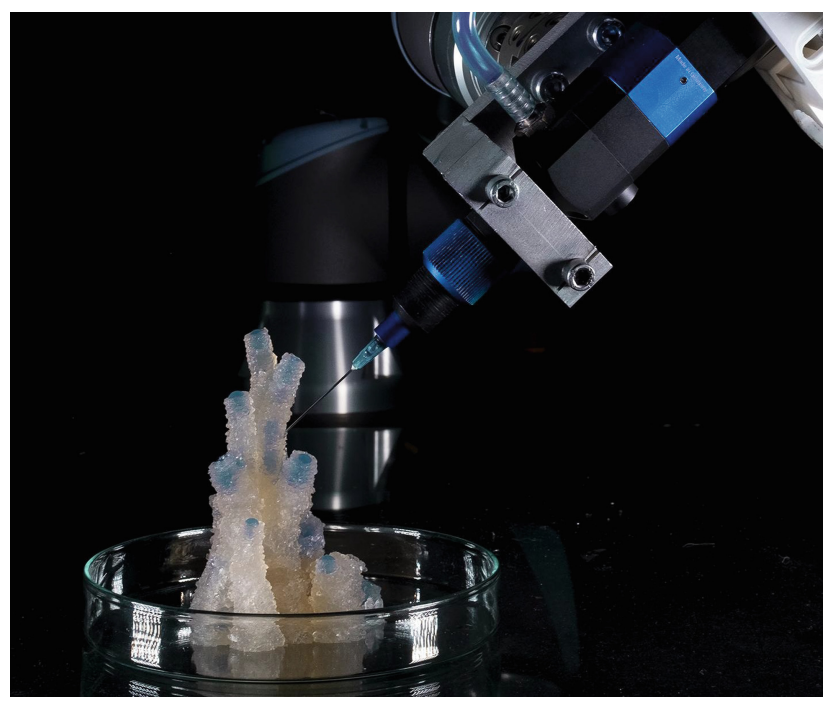

Fig. 1. Micro-architecture of 'Imprimer la Lumière'.

as architectural materiality. By investigating the 3D printing of bioluminescent bacteria, we are questioning how architecture can be host for an ecology of species in symbiotic coexistence (Beesley 2014). Widely occurring in marine life, some mushrooms and insects, bioluminescence in architecture and design remains an under-explored territory of investigation, essentially used to date to imagine and probe more sustainable ways of lighting. In this paper, bioluminescence is used as a mean to question the critical thinking and appropriation of bacteria as an architectural material and how this is changing the practice of architectural design and fabrication.

Our aim is to investigate the critical thinking and appropriation of living bacteria as an architectural materiality. To do so, we explore bioluminescence: a chemical form of light produced by many marine organisms, some insects and mushrooms. While bioluminescent genes are used as markers and for imagining in biology and medicine bioluminescence, recent experiments in the bio-design community have explored bioluminescence as an alternative to public and domestic lighting (Myers 2012; Brayer and Zeitoun 2019). Here, successful experiments have predominantly focused on bioluminescent algae (Van Dongen 2014; Rodriguez 2016, Douenias 2015), we extend this research to bioluminescent bacteria.

In 'Imprimer la Lumière' we use bioluminescent bacteria to examine the metabolism of a living architecture. As any living organism, luminescent bacteria have a limited lifespan. Their appropriation into the built environment therefore induces an intrinsically temporal dimension to the conception, fabrication and experience of architecture, which implies not only the development of a new conceptual framework but also new processes, tools and know-how adapted to microbiologic life that are inseparable from a set of ethical challenges.

As a consequence, this paper asks what are the conceptual, material and technological framework for bio-based architecture and how does designing for living systems 
challenge our current models of design, registration, specification, fabrication and inhabitation. What are the new models by which we can support the design of metabolistic systems of living organisms and capture their limited lifespans and how does living and nurturing become part of a new vocabulary for how we build?

The project is an interdisciplinary collaboration between architecture, design and marine biology undertaken by: CITA (Centre for IT and Architecture, KADK), Soft Matters group, Ensadlab (ENSAD) and Department of Marine Biology (Copenhagen University) (Fig. 2).

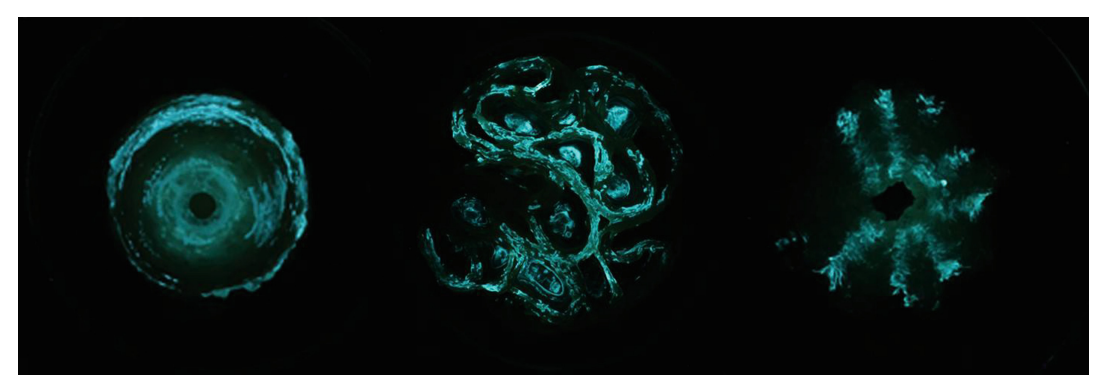

Fig. 2. Micro-architecture performance: the light emitted by the bacteria.

\section{New Methods for a Living Architecture}

'Imprimer la Lumière' presents a set of 3D printed structures acting as a constellation of self-illuminating micro architectures. These micro architectures act as design probes (Ramsgaard Thomsen and Tamke 2009; Mossé 2018) that reflect on the actualisation of architecture as a host for an ecology of species in symbiotic coexistence. The method of the design probe positions the research across conceptual and technological investigations challenging the way we understand the material and performance of contemporary architecture while simultaneously questioning the technologies by which the design and fabrication of living systems can be studied.

Design can be understood in a generic sense as a cultural process of technological appropriation, meaning 'a way of adopting technology in our culture by accepting its influence as well as by influencing it' (de Winter 2002). To question how microbiology can become a new technological platform for architecture, we adopt a practice-based approach informed by the design probe culture (Gaver and Dunne 1999). In this context, a variety of design probes are developed to critically question how to create acceptance for new technological evolutions and discuss whether these changes are desirable. The micro-architectures discussed in this paper sit more specifically at the intersection of conceptual and material probes (Mossé 2018). As a conceptual experiment, they are probed to develop a speculative inquiry or narrative addressing the implications of future desirable cultural patterns. As a material probe, their primary role is to explore the crafts, materials and techniques through which this cultural pattern can be shaped as well their performance as an architectural materiality (Fig. 3). 


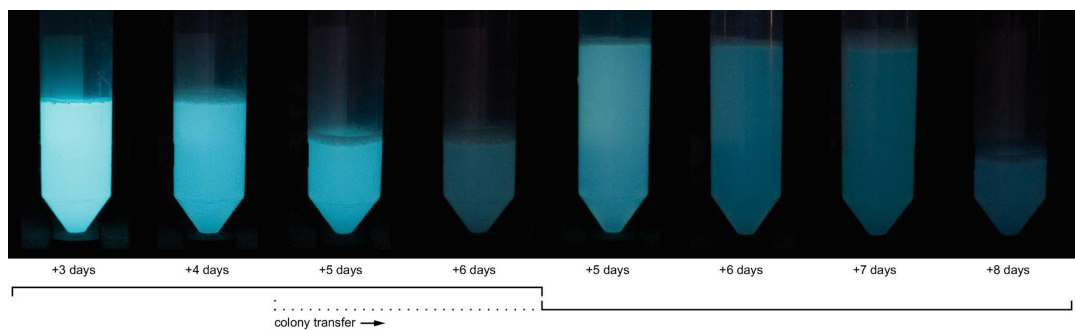

Fig. 3. Vibrio Fischeri cultures in nutritional solution over time from initial activationa.

\section{Developing 'Imprimer La Lumiere’}

The project is conceived over a series of experiments appropriating techniques for growing luminescent bacteria and developing the technologies for 3D printing the extrusion of their medium. 3D printing is explored as a means of liberating the forming processes of the medium to investigate how topology and surface treatment can drive the life cycles and therefore the light performance of the bacteria. In 'Imprimer la Lumière' we use a collaborative robot with a bespoke micro dispenser. This allows us to address an architectural scale of fabrication distinct from dedicated bioprinter that operate at smaller scales. The building of new 3D printing methods for collaborative robots also allows us to interface with programmable design environments, allowing a higher degree of control and steering of both the design and fabrication process.

\subsection{Ethical Considerations}

With biodesign, ethical considerations become an intrinsic part of the design project. Designing with bacteria as a material for architecture means designing for and with the life cycles of living organisms, and allowing co-inhibition within our environment. Where bacteria do die as part of this new architecture, they are also nurtured and invested into a new habitat. In 'Imprimer la Lumière' we work with biosafety level 1 bacteria meaning that they are not genetically modified, do not pose a danger to their surroundings and are fully biodegradable.

In designing with Vibrio Fischeri, we delocalise a marine bacteria to the new host environment of the medium. In doing so we provide a monocultural environment which both optimises its living conditions but also makes it less resilient to change.

\subsection{Designing the Medium for 3D Printing}

The first task is to understand the optimal living environment for the bacteria and design the medium in which it lives. The medium provides nutrition and holds oxygen for the bacteria to metabolise. In 'Imprimer la Lumière' the design of the medium is tailored to the fabrication process and incorporate the requirements for 3D printing. Through experimentation we developed a recipe to control the rheological properties of the medium consisting of Agar, Gelatin, Glycerin, Nutritive media and Water (to 3.2\%, 1.8\%, 2.8\%, $6.5 \%$ respectively). The recipe is developed in order to control the viscosity of the 
medium during the printing process and final state and use for fusion deposition. Agar is used as the basis of the medium for its melting and gelation properties. The amplitude between both temperatures gives the medium durability and firmness (Whistler 1985). Due to this, it is widely used as microbiological media (Dhanapal et al. 2012). We use a common household agar for cooking which enables us to print with additional height, compared to lab grade agarose media otherwise used in direct ink writing technologies (Kilian et al. 2017) (Fig. 4).

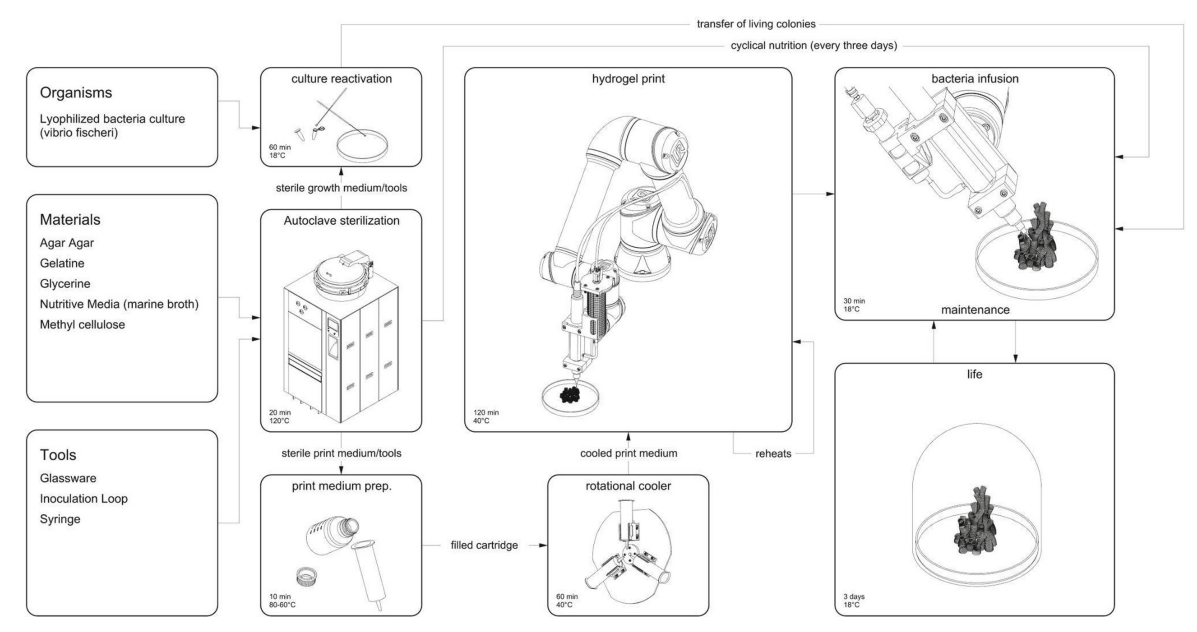

Fig. 4. Fabrication process in 'Imprimer la Lumière'.

In 'Imprimer la Lumière', we require the medium to be kept above the gelation point at all times during printing. To keep the medium cartridge that stores the material at constant temperature before the $3 \mathrm{D}$ printing process, we developed a self-regulating heating unit. Gelatine is added to lower the gelation temperature of the mixture to $40^{\circ} \mathrm{C}$. This reduced temperature conserves energy, enables faster gelation and smooths the surface for a more homogeneous appearance. Additionally, glycerin is used as a plasticizer that increases the flexibility of the intermolecular connections between the Agars polymer chains (Arham et al. 2016). The medium is extruded through a high precision micro-dispensing unit (ViscoTececoPen700) with self-sealing rotor-stator arrangement. The dispensing unit is carried by a 6 axis collaborative robot (UR5e). This allows the robot being used inside laboratory conditions and to be moved to sterile environments for inoculation.

Through this design process, we built a strong understanding of the requirements for the medium and the $3 \mathrm{D}$ printing process. The medium needs to perform as a hybrid of scaffold and nutrition where the containing walls allow dispersion of nutrition and oxygen. During experimentation, we learnt that controlling the surface of the medium was crucial to promote the light-emitting capacity of the cultures. By having a high surface to volume ratio the bacteria is better exposed to oxygen, propagates better and its emitted light becomes easier to perceive. The parallel effort to control the structural 
capacity of the medium in a gelled state allowed us to develop design criteria for the probes as having a large surface, interior cavities and to build high (Fig. 5).
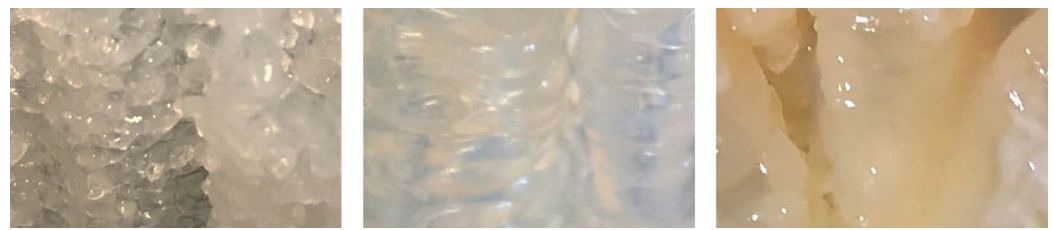

Fig. 5. Changing the composition of medium radically affects the living conditions of the bacteria, the structural capacity and surface quality of the structure

\subsection{Designing a Living Architecture}

The design of the micro-architecture probes employs a differential curve growth algorithm. This allows us to maximise the surface to volume ratio of the structure and optimise the bacteria's light emitting capacity. The line differential growth algorithm also allows us to generate the robot print path as an intrinsic part of the design process allowing greater unity between processes of design and processes of making.

The generative algorithm employs strict form-generating rules to generate complex topologies. In 'Imprimer la Lumière', we adjust the rules to addressing structural and fabrication-based constraints such as maximum overhang, interconnectivity, layer heights and structural performance as a result of wall undulation.

The algorithm follows the logic of differential curve growth. Here, a base curve is iteratively subdivided in selective increments. The curve sections are form found using a physics engine to simulate forces between the subdivision points on the curve. On the subdivision points, sphere colliders force these segments to elongate, resulting in the differential growth of the geometry.

In order to achieve a suitable degree of surface complexity and to ensure a balanced distribution of complexity, we employ the differential growth algorithm in two stages: firstly to generate a satisfactory base geometry and secondly to grow the topology in three dimensions. Narrow passages, emerging from the generative process are detached allowing the probe geometry to branch. The splitting is conditioned by a straight skeleton calculation evaluating local surface area. The single branches are then calculated within the incremental growth process as individual objects within a unified system. This process ensures that the overall forces driving the form finding process apply equally to the topological whole and that structural and fabrication requirements are fulfilled. The process can be locally varied to account for structural support and gravity.

The branching structure generates a complex interior section with distributed hollows that act as vessels for bacterial growth. The complexity of the micro-architecture probes allow us to steer the emission of light through topology. 


\subsection{The Inoculation Process}

Lifecycle optimisation and the strict environmental requirements of the cultures demand new fabrication processes bringing new challenges and opportunities (Dade-Robertson 2019; Colette 2007; Pasquero and Poletto 2014). In 'Imprimer la Lumière', the manufacturing process requires the nurturing of the bacteria culture and its shielding from impending contamination. Activated from lyophilized state, the culture is pre-grown within nutritive media suspension while the printed probe is inoculated with a microdispenser. This allowed for stronger recognisable glow, which can be attributed to the reintroduction of oxygen through diffusion. The inoculation process uses the robot for precise positioning. A digital model of the probe is used to compute the access-path for the robot. The probe is then injected with the bacteria suspension and filled into its vessels (Fig. 6).
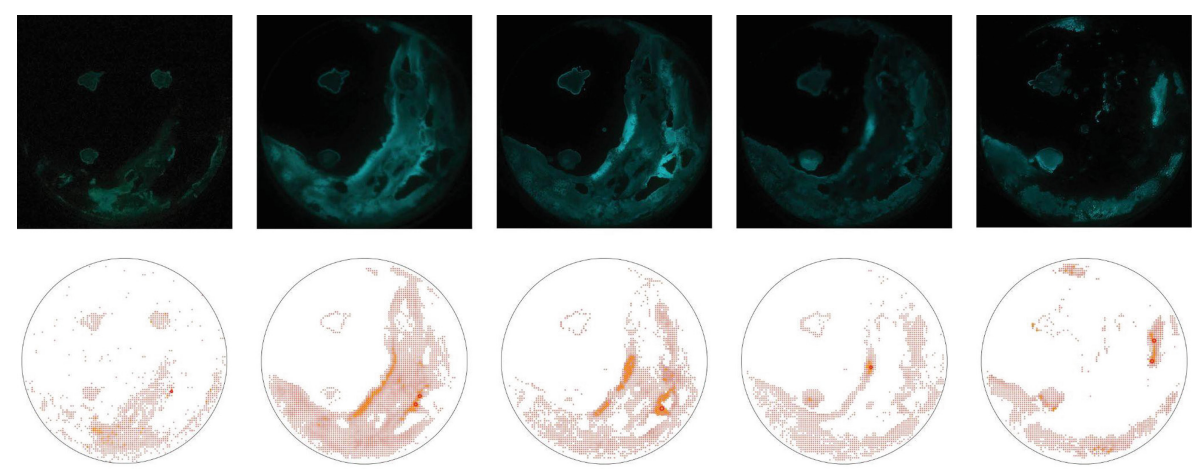

Fig. 6. Bacteria culture growing on medium over time with luminescence analysis.

During tests we observed an activation time of an average of three days. For the colony to survive, a sterile environment containing sufficient oxygen and nutrition needs to be ensured. Prior experiments have shown that the gelation of the agar prohibits diffusion of additional oxygen, which renders active air exchange obsolete. To understand the life cycle of the probes, the timeframe of the culture's growth and propagation needs to be considered.

\subsection{Evaluation}

The probes are evaluated using two processes of observation and oxygen sensing. The first process uses imaging to register the bacteria growth and propagation patterns across the surface in time. The images are taken in the dark to allow best understanding of the bacteria's light emitting properties. The colony is observed across 12 days in which we observe firstly the growth of the colony around the inoculation point and the strengthening of the light emitted (day 2-4) and secondly a move of the light emitted away from the inoculation point (day 5-12). Our assumption is that the colony propagates into new medium as nutrition and oxygen is consumed. To test this assumption a second evaluation 
is undertaken using optical oxygen sensing based on the dynamic quenching of an oxygen sensitive indicator dye by oxygen. Here, an optical oxygen meter (FireStingO2) with a retractable needle-type sensor (OXR230) from Pyroscience (pyroscience.com) is used for measurements of dissolved oxygen. Oxygen is measured at the surface of the bacterial culture and different depths through the culture and the medium towards the bottom of the petri-dish. Additional measurements are done at the interface of the bacterial culture and medium, and stepwise further away from the bacterial culture at approximately $1 \mathrm{~mm}$ depth within the medium. A clear gradient in oxygen concentration became apparent, as oxygen depleted gradually from the medium (approx. 100\% air saturation) towards the bacterial culture, which was completely anoxic ( $0 \%$ air saturation). An oxygen profile measured from the surface of the culture through the medium to the bottom of the petri-dish showed similar results. The bacterial culture proved to be anoxic, and the medium below increased in oxygen concentration the further away from the bacteria measurements were conducted (Fig. 7).
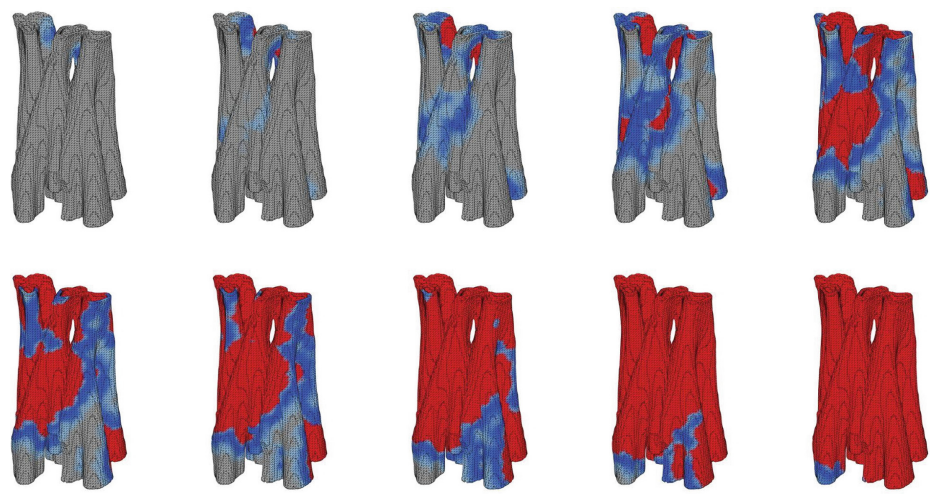

Fig. 7. Particle system simulating the progress of bacteria propagation through the medium.

\subsection{Simulation of Bacteria Behaviour}

In order to understand the lifecycle of the bacteria, we develop a simulation of propagation through the medium. Herefore, we use a simple particle engine to approximate the printed geometry. These are equipped with a density attribute computed by the proximity to their neighbours. The particles closest to the inoculation points are activated as live particles. This attribute is used to control and call the propagation of neighbouring particles by counting up a 'life-time' attribute across each time-step of the calculation. Neighboring particles are then 'activated' in subsequent iterations. When a particles 'lifetime' surpasses a defined maximum value, it transitions into a 'dead' particles, simulating the state where all oxygen and nutrition is consumed. No additional particles can be activated from this point. The simulation of propagation speed and lifespace is calibrated using the observed imagining data from the physical probes. 


\section{Conclusion}

'Imprimer la Lumière' sits at the intersection of biodesign and digital fabrication. It opens up new perspectives for the design and appropriation of bioluminescence as an architectural materiality. At a conceptual level, the project asks what happens when the material of architecture becomes living: what are the new concepts, methods and technologies that are needed to design for and with living materials?

The first step in 'Imprimer la Lumière' is to link bio-design agendas with digital fabrication and 3D printing. Our application of collaborative robots for 3D printing of microbiologic organism allow us to extend the scale of fabrication and speculate upon the design criteria. In 'Imprimer la Lumière', the probes are informed by both the optimal living environment for the bacteria and the fabrication criteria for 3D printing. By controlling the rheological properties of the medium and its gelling, we can steer the light-emitting performance and design its structural capability to build complex topologies with interior cavities and vessels that optimise the living environment for the bacteria.

The second step is to build assumptions about how the geometry of the microarchitecture affects performance. These assumptions are made through empirical observation studies and evaluated through localised oxygen sensing. The assumptions are used as a basis to build prototypical models that can simulate and capture the light emitting performance and propagation of the bacteria as it moves through the medium, 'Imprimer la Lumière' speculates on what the nature of futures representations can be. Where traditional architectural representation emphasises the description of form through the geometry of extension, 'Imprimer la Lumière' develops volumetric models that change across time steps. These descriptions allow architects and designers to specify and describe the design and steering of material lifecycles and their associated performances.

The probes in 'Imprimer la Lumière' look like towers or dense cities in small scale. Conceived as micro-architectures, their aim is not to solve an architectural performance such as public or domestic lighting, but instead to probe what a living architecture could mean.

Acknowledgment. 'Imprimer la lumière' is a cross-disciplinary collaboration between CITA (Centre for IT and Architecture, KADK), Soft Matters group, Ensadlab (ENSAD) and Department of Marine Biology (Copenhagen University). The project has benefited from the support of the IFD sciences programme of Institut Français du Danemark (2018) and subsequent funding from the Danish Arts Foundation (2019). We thank Leuchtlabor GbR, Weiherhammer, Germany for their kind support. We thank the students of CITA Computation in Architecture (Aroni Roy,Tessira Reyes Crawford, Claudia Colmo, Izabella Banas, Kawtar Al Akel, Ke Lin, Nikhila Vedula, Youcheng Li, Carolin Feldmann) for their contribution to observational studies. 


\section{References}

Arham, R., Mulyati, M., Metusalach, M., Salengke, S.: Physical and mechanical properties of agar based edible film with glycerol plasticizer. Int. Food. Res. J. 23(4), 1669-1675 (2016)

Beesley, P.: Dissipative models: notes toward design method, paradigms in computing: making, machines and models for design agency in architecture. Evolo, 24-34 (2014)

Brayer, M.A., Zeitoun, O.: La Fabrique du Vivant: Mutations Créations. HYX, Paris (2019)

Colette, C.: BioLace: an exploration of the potential of synthetic biology and living technology for future textiles. Stud. Mater. Think. 7, 1-14 (2007)

Dade-Robertson, M.: The designs of the natural. In: Bühlmann, V. (ed.) Ghosts of Transparency: Shadows Cast and Shadows Cast Out, pp. 197-206. De Gruyter, Berlin, Boston (2020)

Dhanapal, D., Sasikala, P., Rajamani, L., Kavitha, V., Yazhini, G., Shakila Banu, M.: Edible films from Polysaccharides. Food Sci. Qual. Manage. 3, 9-18 (2012)

VanDongen, T.: Ambio (2014). (Van Dongen, T.: (2019))

Franklin, K., Till, C.: Radical Matter: Rethinking Materials for a Sustainable Future. Thames \& Hudson, London (2018)

Gaver, W.W., Dunne, A.: Cultural probes. Interactions 6(1), 21-29 (1999)

Kilian, D., Ahlfeld, T., Akkineni, A.R., Lode, A., Gelinsky, M.: Three-dimensional bioprinting of volumetric tissues and organs. MRS Bull. 42(8), 585-592 (2017). https://doi.org/10.1557/mrs. 2017.164

Mossé, A.: Gossamer timescapes: a design-led investigation into electro-active and lightresponsive textiles for the home. Smart Mater. Struct. 27(7), 24-34 (2018)

Myers, W.: Biodesign. Thames \& Hudson, London (2012)

Pascero, C., Poletto, M.: Ecology beyond nature. In: Walter de Gruyter, M., Hovestadt, L. (eds.) Alive: Advancements in Adaptive Architecture, Walter de Gruyter GmbH, Zurich (2012)

Terranova, C.N., Tromble, M. (eds.): The Routledge Companion to Biology in Art and Architecture. Routledge, United-Kingdom (2017)

Ramsgaard Thomsen, M., Tamke, M.: Narratives of making: thinking practice led research in architecture, Communicating (by) Design, Bruxelles, pp. 1-8 (2009)

Ramsgaard Thomsen, M., Tamke, M., Nicholas, P., Svilans, T.: A new material vision. In: Diniz, N. (ed.) Data: Matter, ACTAR Press (2020)

Whistler, R.L., Daniel, J.R.: Carbohydrates. In: Fennema, O.R. (ed.), Food Chemistry, pp. 69-137. Marcel Dekker, New York (1985)

de Winter, K.: Thoughts on originality (2002). https://medium.com/age-of-awareness/the-end-oforiginality-51f79aacd9ac 
Open Access This chapter is licensed under the terms of the Creative Commons Attribution 4.0 International License (http://creativecommons.org/licenses/by/4.0/), which permits use, sharing, adaptation, distribution and reproduction in any medium or format, as long as you give appropriate credit to the original author(s) and the source, provide a link to the Creative Commons license and indicate if changes were made.

The images or other third party material in this chapter are included in the chapter's Creative Commons license, unless indicated otherwise in a credit line to the material. If material is not included in the chapter's Creative Commons license and your intended use is not permitted by statutory regulation or exceeds the permitted use, you will need to obtain permission directly from the copyright holder. 